\title{
A Deep Study of the 3C 273 Field in $\gamma$-rays
}

\author{
I. Brott ${ }^{1,2}$, I. Kreykenbohm ${ }^{1,3}$, P. Lubinski ${ }^{4,1}$, N. Produit ${ }^{1}$, \\ P. H. Hauschildt ${ }^{2}$, and T. J.-L.Courvoisier ${ }^{1}$ \\ ${ }^{1}$ INTEGRAL Science Data Centre, Chemin d'Ecogia 16, 1290 Versoix, Switzerland \\ e-mail : Ines.Brott@obs.unige.ch \\ ${ }^{2}$ Hamburger Sternwarte, Gojenbergsweg 112, 21029 Hamburg, Germany \\ ${ }^{3}$ Institut für Astronomie und Astrophysik - Astronomie, Sand 1, 72076 Tübingen, Germany \\ ${ }^{4}$ N. Copernicus Astronomical Center, Bartycke 18, 00-716 Warszene, Poland
}

\begin{abstract}
C 273 is one of the brightest and best studied quasars. It has been observed for 770 ks with the imager IBIS (FoV $12 \mathrm{deg}$ ) on board INTEGRAL. To achieve the best possible $\mathrm{S} / \mathrm{N}$ the dataset has been screened using several criteria indicating the quality of the data (i.e., number of good time intervals, etc). We describe the necessary tools and methods to analyze data of deep fields.
\end{abstract}

Keywords. galaxies: active, gamma rays: observations, methods: data analysis.

\section{Introduction}

Low sensitivity of former missions has prevented a detailed study of the hard cosmic X-ray background. As in soft X-rays it has been resolved to AGN (Giacconi et al. 2002), a similar situation is anticipated for soft $\gamma$-rays (Worsley et al. 2004; Krivonos et al. 2005). 3C 273 has been observed regularly twice a year by INTEGRAL since 2003. We have used all data available to us (June 2005) within $15^{\circ}$ of $3 \mathrm{C} 273$ between revolutions 28 and 207 with a total exposure time of $774 \mathrm{ks}$ in 371 science windows $(\mathrm{ScW})$.

\section{Data Analysis and Results}

To avoid instrumental background lines which are located between $59.3 \mathrm{keV}$ and $84.9 \mathrm{keV}$ ( $\mathrm{W}$ and $\mathrm{Pb}$ fluorescence) and a blend of several flurocence lines of $\mathrm{Cd}$ and Te around $24 \mathrm{keV}$ (Terrier et al. 2003), we selected a band from $26-51 \mathrm{keV}$ for our analysis, while using data from $13-26 \mathrm{keV}$ and $51-105 \mathrm{keV}$ to assess the background level. Data reduction was done with OSA 5 as distributed by the ISDC. We rejected ScWs due to: unacceptable artefacts in the deconvolved image (17), average good time interval less than 10 seconds long (6), and an unusual background level if in 2 of 3 energy bands $\left|\xi_{i}-\chi\right|>\frac{1}{N} \sum_{i}\left|\xi_{i}-\chi\right|(53 \mathrm{ScWs}) . \xi_{i}$ denotes the median pixel countrate in the shadowgram and $\chi$ the median over those $\xi_{i}$, the index $i$ is running over the ScWs. In total 66 ScWs were excluded, mostly from revolution 28-32 when the bottom veto shield of IBIS was turned off (see Fig. 1).

Due to the dithering strategy the exposure time varies in the mosaic from $633 \mathrm{~s}$ to $838 \mathrm{ks}$, therefore the source detection was limited to a field of $\sim 1800 \mathrm{deg}^{2}$ for which $T_{\exp }>150 \mathrm{ks}$. In order to emphasize source signal with respect to the background we convolved the field with a gaussian core and searched for the local maxima in the folded image. The FWHM of the gaussian core was calibrated on 3C 273. We searched for sources with a core volume larger than 4.5 and round in shape. 

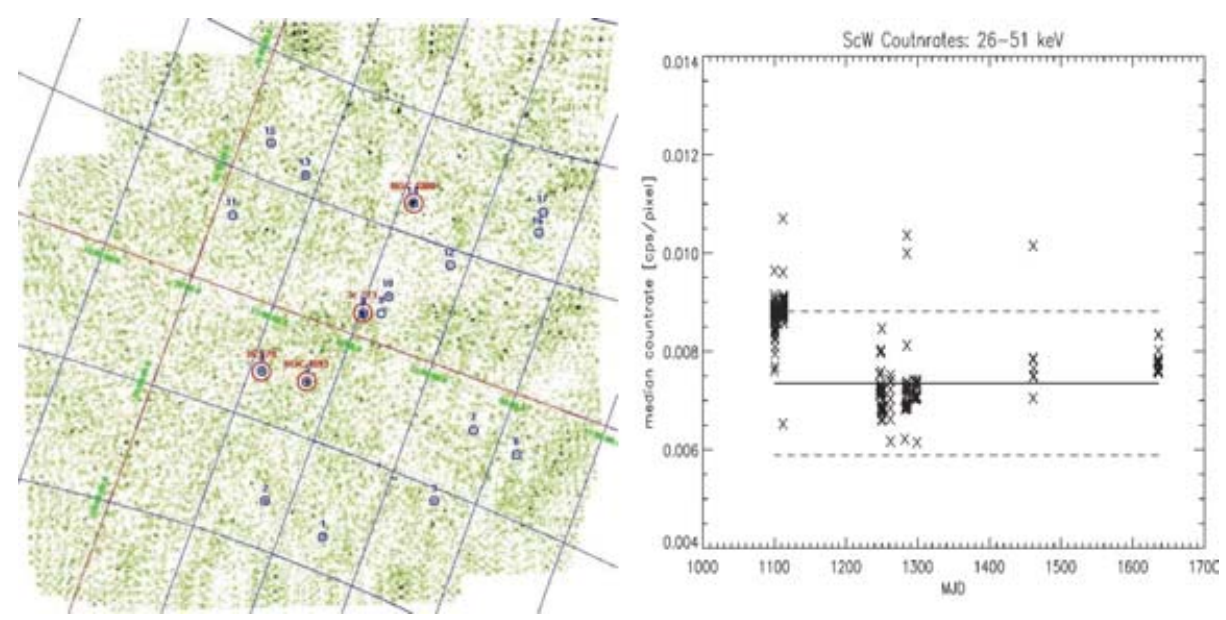

Figure 1. Left: S/N image of the 3C 273 field. Right: Evolution of the background with time.

\begin{tabular}{|c|c|c|c|c|c|c|}
\hline \# & Ra & | Dec & | Possible Identifier & $\Delta \operatorname{Pos}$ & |S/N & Type \\
\hline $1 *$ & $\mid \begin{array}{ll}12 & 1518\end{array}$ & $\mid \begin{array}{lll}-17 & 17 & 19\end{array}$ & - & - & 4.63 & - \\
\hline $2 *$ & 123903 & -160827 & XSS J12389-1614 & $5.9^{\prime}$ & .63 & $\mathrm{X}$ \\
\hline 3 & 114332 & -110357 & - & - & 4.81 & - \\
\hline $4 *$ & 125609 & -054658 & $3 \mathrm{C} 27$ & 0.6 & 5.88 & QSO \\
\hline $5 *$ & 123940 & -051933 & NGC 4593 & $1.1^{\prime}$ & 20.44 & Sy2 \\
\hline 6 & 112320 & -044842 & & 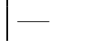 & 4.24 & \\
\hline 7 & 11 & 1129 & $\mathrm{AC}$ & 1.9 ' & 7.5 & $\mathrm{GiC}$ \\
\hline $8 *$ & 12 & 214 & $3 \mathrm{C}$ & - & 37.05 & QSO \\
\hline $9 *$ & 122318 & +023812 & Mrk & 2.8 & 2.99 & Sy1 \\
\hline $10 *$ & 122232 & +041810 & NGC 4303 & 10.3 & 4.62 & Sy2 \\
\hline 11 & 132422 & +061225 & - & — & 4.17 & - \\
\hline 12 & 12 & +083745 & - & - & 3.51 & - \\
\hline 13 & 1 & 142 & - & - & 5.81 & - \\
\hline $14^{*}$ & 12 & 738 & NG & $2.5^{\prime}$ & 39.08 & Sy2 \\
\hline $15^{*}$ & 132028 & +130734 & 1RXS J132036. & $8.1^{\prime}$ & 4.64 & $\mathrm{X}$ \\
\hline 16 & 114117 & +132926 & - & - & 3.91 & - \\
\hline 17 & 114203 & +151752 & — & - & 4.50 & - \\
\hline
\end{tabular}

Table 1. Counterparts have been searched within 10' in SIMBAD. '*' denotes detections that have been found in the extended $26-105 \mathrm{keV}$ band as well.

To assess the validity of these detections we performed the same search in the joined 26-105 keV mosaic, resulting in 24 detections, 9 coincident with detections from the 26$51 \mathrm{keV}$ band. A search for negative excesses in the $26-51 \mathrm{keV}$ band resulted in 10 detections. Therefore it is possible that most of the non identified sources are false detections.

This preliminary analysis shows that a detection of weak sources is possible, but the statistics are low and the weak sources are highly background dominated. With the availability of more data on this field these faint sources can be studied in more detail.

\section{References}

Giacconi, R., Zirm, A., Wang, J., et al. 2002, ApJS, 139, 369

Krivonos, R., Vikhlinin, A., Churazov, E., et al. 2005, ApJ, 625, 89

Terrier, R., Lebrun, F., Bazzano, A., et al. 2003, A\&A, 411, L167

Worsley, M. A., Fabian, A. C., Barcons, X., et al. 2004, MNRAS, 352, L28 